\title{
Theory and Practice of Diffractometry on Single Tungsten Atoms using Electron Microscope Pixel Array Detectors
}

Michael C. Cao ${ }^{1}$, Yimo Han ${ }^{1}$, Yi Jiang ${ }^{2}$, Kayla X Nguyen ${ }^{3}$, Prafull Purohit ${ }^{4}$, Mark W. Tate ${ }^{4}$, Sol M Gruner $^{2,4,5,6}$, Veit Elser ${ }^{2}$, David A. Muller ${ }^{1,5}$

1. School of Applied and Engineering Physics, Cornell University, Ithaca, NY, USA

2. Department of Physics, Cornell University, Ithaca, NY, USA

3. Department of Chemistry and Chemical Biology, Cornell University, Ithaca, NY, USA

4. Laboratory of Atomic and Solid State Physics, Cornell University, Ithaca, NY, USA

5. Kavli Institute for Nanoscale Science, Cornell University, Ithaca, NY, USA

${ }^{6 .}$ Cornell High Energy Synchrotron Source, Cornell University, Ithaca, NY, USA

A new generation of pixelated detectors has expanded the possibility for collecting full scattering information by recording a full diffraction pattern at every scanning point, creating a 4D dataset where a rich variety of physical information can be extracted [1,2]. To properly collect all scattering information, our group has developed the Electron Microscope Pixel Array Detector (EMPAD), which is capable of 1,000,000:1 dynamic range with single electron sensitivity and millisecond readout time for scanning transmission electron microscopy [2]. Analysis of these full and unsaturated diffraction patterns allow for a wide variety of new quantitative imaging modes with direct physical meaning.

One such imaging mode is the Center of Mass (COM) which measures the expectation value of the inplane momentum shift $\langle\mathrm{p}\rangle$ to give the probability current flow and retrieves differential phase contrast (DPC) [3,4]. Using a phase object model, this is interpreted as the gradient of the potential, but the momentum distribution is apparently different for classical and quantum limits. Classically, the electron beam is attracted to the nucleus, resulting in a shifted bright disk in the diffraction pattern. A split quadrant detector measures this shift, creating a conventional DPC image. We confirm this, but only in the asymptotic limit where the probe size is much smaller than the feature size, typically seen in a sample with a slowly-varying field.

However, atomic-resolution imaging is outside this limit, and the bright disk remains unshifted during scan. Instead, intensity within the stationary disk is shifted due to the attraction of the electron beam to the atom column. Rather than an asymmetry in the placement of the bright disk, there is an asymmetry in the bright disk itself, measured by a "first moment" detector, creating the COM image. This can be interpreted as a bias in the $\mathrm{e}^{-}$probability current flow towards the positive charged nucleus (figure 1). Tracking only the shift of the disk, and not its inner details has the effect of low-pass filtering an image, leaving only those features much larger than the probe itself, irrespective of their origin.

In real space, the COM image is a cross-correlation of the probe shape and the potential gradient, which does not accurately represent the sample potential gradient in the large probe size regime. Instead, the sample potential tends to a delta function and the COM signal becomes the inverted, reflected gradient of the probe (Figure 2). While the intensity of the signal is linear with the strength of the sample potential, the image profile is shaped by the probe itself rather than the sample potential.

Considerations of detector geometry and its effects on image coherence and signal to noise ratio (SNR) are also investigated. Simulations of SNR for a COM image of a carbon atom at $60 \mathrm{keV}$ show significant 
gains in SNR when integrating near the edge of the aperture. Additionally, an analytic expression is given to describe the coherence/incoherence of an image for any given detector geometry [5].

\section{References:}

[1] V. B. Ozdol et al. Applied Physics Letters 106 (2015), p. 253107

[2] M. W. Tate et al. Microscopy and Microanalysis 22 (2016), p. 237-249.

[3] A. Lubk, J. Zweck, Physical Review A 91 (2015), p. 023805.

[4] I. Lazić, E.G.T. Bosch, S. Lazar, Ultramicroscopy 160 (2016), p. 265-280.

[5] The authors acknowledge funding from the DOD/AFOSR 2D MURI project and facilities support from the Cornell Center for Materials Research, an NSF MRSEC (DMR-1120296).
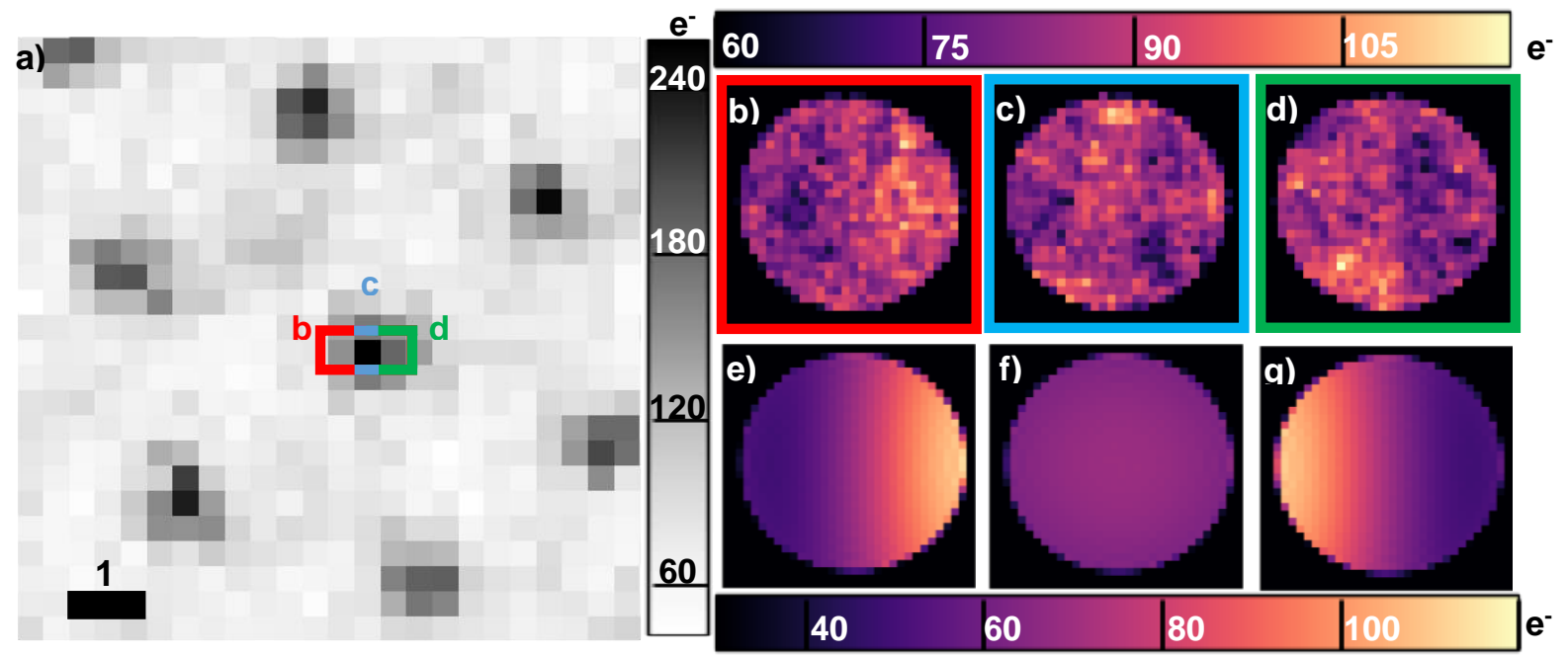

Figure 1. a) HAADF image constructed from experimental diffraction data collected on $\mathrm{WSe}_{2}$ at $120 \mathrm{keV}$ with a 30 mrad aperture. b,c,d) Diffraction patterns scanning left to right over the boxed tungsten atom in a). The asymmetrical intensity in the bright disk in b) and d) show the electron beam's attraction to the atom. e,f,g) Simulated diffraction patterns predicting the asymmetrical intensity shift in the bright disk.
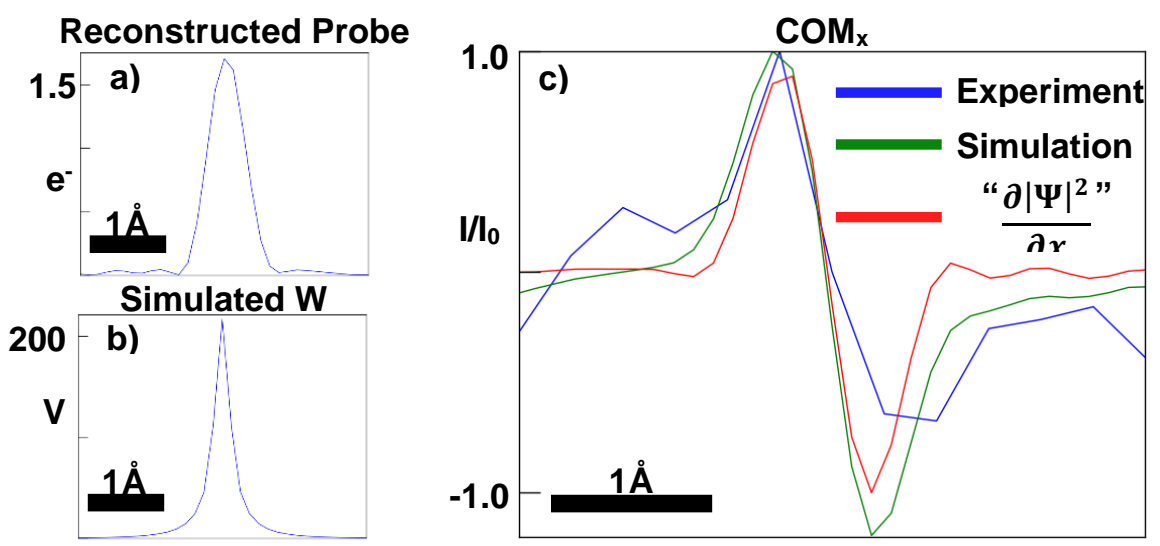

Figure 2. a) Profile of the probe reconstructed from the same $\mathrm{WSe}_{2}$ data in Fig. 1 using ptychography. b) Simulated potential of the tungsten atom shows that the probe width is larger than the potential. c) Normalized comparisons of the $\mathrm{COM}_{\mathrm{x}}$ line profile across the tungsten atom shown in Fig. 1a, the simulated profile using the tungsten potential in $b$ ), and the gradient of the reconstructed probe shows agreement that for a small feature size, the resulting profile matches the gradient of the probe rather than the potential. Rather than probing the sample, the shape of the signal samples the probe. 